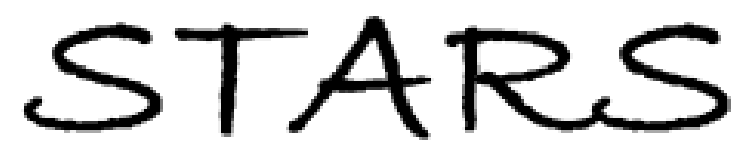

University of Central Florida

STARS

$1-1-2008$

\title{
First Confirmed Detection of a Bipolar Molecular Outflow From A Young Brown Dwarf
}

Ngoc Phan-Bao

Basmah Riaz

Chin-Fei Lee

Ya-Wen Tang

Paul T. P. Ho

See next page for additional authors

Find similar works at: https://stars.library.ucf.edu/facultybib2000

University of Central Florida Libraries http://library.ucf.edu

This Article is brought to you for free and open access by the Faculty Bibliography at STARS. It has been accepted for inclusion in Faculty Bibliography 2000s by an authorized administrator of STARS. For more information, please contactSTARS@ucf.edu.

\section{Recommended Citation}

Phan-Bao, Ngoc; Riaz, Basmah; Lee, Chin-Fei; Tang, Ya-Wen; Ho, Paul T. P.; Martin, Eduardo L.; Lim, Jeremy; Ohashi, Nagayoshi; and Shnag, Hsien, "First Confirmed Detection of a Bipolar Molecular Outflow From A Young Brown Dwarf" (2008). Faculty Bibliography 2000s. 840.

https://stars.library.ucf.edu/facultybib2000/840

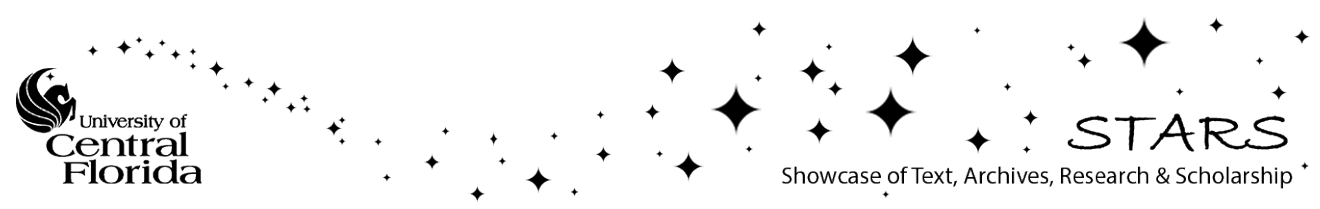




\section{Authors}

Ngoc Phan-Bao, Basmah Riaz, Chin-Fei Lee, Ya-Wen Tang, Paul T. P. Ho, Eduardo L. Martin, Jeremy Lim, Nagayoshi Ohashi, and Hsien Shnag 
The Astrophysical Journal, 689: L141-L144, 2008 December 20

(C) 2008. The American Astronomical Society. All rights reserved. Printed in U.S.A.

\title{
FIRST CONFIRMED DETECTION OF A BIPOLAR MOLECULAR OUTFLOW FROM A YOUNG BROWN DWARF \\ Ngoc Phan-Bao, ${ }^{1}$ Basmah Riaz, ${ }^{2}$ Chin-Fei Lee, ${ }^{1}$ Ya-Wen Tang, ${ }^{1}$ Paul T. P. Ho, ${ }^{1,3}$ Eduardo L. Martín, ${ }^{2,4}$ Jeremy Lim, ${ }^{1}$ Nagayoshi Ohashi, ${ }^{1}$ and Hsien Shang ${ }^{1}$ \\ Received 2008 September 25; accepted 2008 October 29; published 2008 November 7
}

\begin{abstract}
Studying the earliest stages in the birth of stars is crucial for understanding how they form. Brown dwarfs with masses between that of stars and planets are not massive enough to maintain stable hydrogen-burning fusion reactions during most of their lifetime. Their origins are subject to much debate in recent literature because their masses are far below the typical mass where core collapse is expected to occur. We present the first confirmed evidence that brown dwarfs undergo a phase of molecular outflow that is typical of young stars. Using the Submillimeter Array, we have obtained a map of a bipolar molecular outflow from a young brown dwarf. We estimate an outflow mass of $1.6 \times 10^{-4} M_{\odot}$ and a mass-loss rate of $1.4 \times 10^{-9} M_{\odot}$. These values are over 2 orders of magnitude smaller than the typical ones for $\mathrm{T}$ Tauri stars. From our millimeter continuum data and our own analysis of Spitzer infrared photometry, we estimate that the brown dwarf has a disk with a mass of $8 \times$ $10^{-3} M_{\odot}$ and an outer disk radius of $80 \mathrm{AU}$. Our results demonstrate that the bipolar molecular outflow operates down to planetary masses, occurring in brown dwarfs as a scaled-down version of the universal process seen in young stars.
\end{abstract}

Subject headings: ISM: individual (ISO-Oph 102, [GY92] 204) — ISM: jets and outflows stars: formation - stars: low-mass, brown dwarfs — techniques: interferometric

\section{INTRODUCTION}

Star formation starts with collapse, accretion, and launching of material as a bipolar outflow (Lada 1985). It is thought that brown dwarfs could undergo the same stages of formation as stars and that they have a common origin (Luhman et al. 2007). However, the typical masses of brown dwarfs, 15-75 $M_{\mathrm{J}}$, are far below the typical Jeans mass in molecular clouds, and hence it is difficult to make a large number of them by direct gravitational collapse. Several brown dwarf formation mechanisms have been proposed (see Whitworth et al. 2007 and references therein), and more observations are needed to improve our understanding of the role of each of them.

Recent observational evidences for accretion and optical jets have been found in young brown dwarfs (Martín et al. 2001; Fernández \& Comerón 2001; Jayawardhana et al. 2003; Whelan et al. 2007). However, there is no firm evidence up to now to demonstrate that the brown dwarf formation involves the bipolar molecular outflow process as seen in young stars. In the case of low-mass stars, the molecular outflow plays a vital role (Bachiller \& Gómez-González 1992; Bachiller 1996; Reipurth \& Bally 2001; McKee \& Ostriker 2007) to testing the lowmass star formation theory (Shu et al. 1991; Masson \& Chernin 1993; Raga \& Cabrit 1993; Pudritz et al. 2007). Therefore, the detection of molecular outflows provides a key piece of information on the earliest phase of the brown dwarf formation and allows us to constrain the low-mass star formation theory in the substellar domain.

In this Letter, we present millimeter observations of the young brown dwarf ISO-Oph 102 in the $\rho$ Ophiuchi dark cloud. We detect a bipolar molecular outflow from the brown dwarf, demonstrating that this outflow mechanism as seen in young

\footnotetext{
${ }^{1}$ Institute of Astronomy and Astrophysics, Academia Sinica, P.O. Box 23141, Taipei 106, Taiwan, China; pbngoc@asiaa.sinica.edu.tw.

${ }^{2}$ Instituto de Astrofísica de Canarias, C/ Vía Láctea s/n, E-38200 La Laguna, Tenerife, Spain.

${ }^{3}$ Harvard-Smithsonian Center for Astrophysics, Cambridge, MA 02138.

${ }^{4}$ University of Central Florida, Department of Physics, P.O. Box 162385, Orlando, FL 32816-2385.
}

stars continues to operate down to the masses of brown dwarfs. We show that the outflow mass is much smaller than typical values found in low-mass stars by 2-3 orders of magnitude. We also combine our radio data with infrared data to estimate disk parameters. The infrared spectrum of ISO-Oph 102 shows strong crystalline silicate features. This demonstrates dust processing in the protoplanetary disk (Apai et al. 2005) of the brown dwarf. The coexistence of the molecular outflow with the crystallization of dust grains could be of importance for models of planet formation.

Section 2 presents the millimeter observations and the data reduction, $\S 3$ discusses the physical properties of the bipolar molecular outflow, the disk parameters, and the crystalline silicate features in ISO-Oph 102, and $\S 4$ summarizes our results.

\section{OBSERVATIONS AND DATA REDUCTION}

ISO-Oph 102 (or [GY92] 204), a young M6 dwarf initially identified by Greene \& Young (1992), is located in the $\rho$ Ophiuchi dark cloud at a distance of 125 pc (de Geus et al. 1989). Its estimated mass is $60 M_{\mathrm{J}}$ (Natta et al. 2002). This value is below the hydrogen-burning limit and in good agreement with the dynamically measured mass of an M6.5 dwarf of $55 M_{\mathrm{J}}$ at the same age (see Stassun et al. 2005). ISO-Oph 102 is therefore a young bona fide brown dwarf. The presence of lithium absorption in its optical spectrum (Natta et al. 2004) supports its brown dwarf nature (Martín et al. 1994). Strong evidence for the presence of an accretion disk has been reported (Natta et al. 2004). The blueshifted optical jet component was discovered by Whelan et al. (2005). The optical visibility of ISO-Oph 102 suggests that the source corresponds to a class II object (Lada 1985) in the star formation phase; a class with an accreting circumstellar disk and the protostar at this stage is the so-called classical T Tauri star.

We have observed ISO-Oph 102 with the receiver band at 


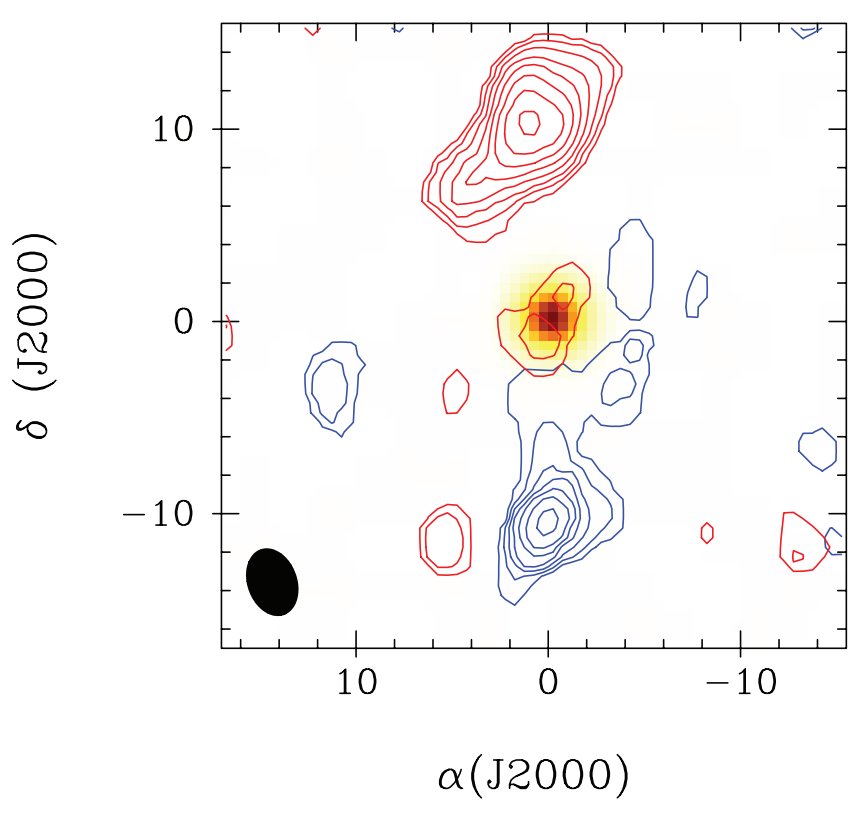

FIG. 1.-Overlay of the $J$-band $(1.25 \mu \mathrm{m})$ near-infrared Two Micron All Sky Survey (2MASS) image and the integrated intensity in the carbon monoxide (CO $J=2-1$ ) line emission from 3.8 to $7.7 \mathrm{~km} \mathrm{~s}^{-1}$ line-of-sight velocities. The blue and red contours represent the blueshifted (integrated over 3.8 and $5.9 \mathrm{~km} \mathrm{~s}^{-1}$ ) and redshifted (integrated over 5.9 and $7.7 \mathrm{~km} \mathrm{~s}^{-1}$ ) emissions, respectively. The contours are $3,6,9, \ldots$ times the rms of $0.15 \mathrm{Jy}$ beam $^{-1} \mathrm{~km} \mathrm{~s}^{-1}$. The brown dwarf is visible in the $J$-band image. The position angle of the outflow is about $3^{\circ}$. The peaks of the blue- and redshifted components are symmetric to the center of the brown dwarf with an offset of $10^{\prime \prime}$. The synthesized beam is shown in the bottom left corner.

$230 \mathrm{GHz}$ of the $\mathrm{SMA}^{5}$ (Ho et al. 2004). Both $2 \mathrm{GHz}$-wide sidebands, which are separated by $10 \mathrm{GHz}$, were used. The SMA correlator was configured with high spectral resolution bands of 512 channels per chunk of $104 \mathrm{MHz}$ for ${ }^{12} \mathrm{CO},{ }^{13} \mathrm{CO}$, and $\mathrm{C}^{18} \mathrm{O} J=2 \rightarrow 1$ lines, giving a channel spacing of 0.27 $\mathrm{km} \mathrm{s}^{-1}$. A lower resolution of $3.25 \mathrm{MHz}$ per channel was set up for the remainder of each sideband. The quasars 1625-254 and 3C 279 have been observed for gain and passband calibration, respectively. Mars was used for flux calibration. The data were calibrated using the MIR software package, and further analysis was carried out with the MIRIAD package adapted for the SMA. The compact configuration was used, resulting in a synthesized beam of $3.60^{\prime \prime} \times 2.43^{\prime \prime}$ with a position angle of $8.5^{\circ}$. The rms sensitivity was about $3 \mathrm{mJy}$ for the continuum, using both sidebands and $\sim 0.2 \mathrm{Jy} \mathrm{beam}^{-1}$ per channel for the line data. The primary FWHM beam is about $55^{\prime \prime}$ at the observed frequencies. The integrated flux density from the dust continuum emission was $7 \pm 3 \mathrm{mJy}$ measured at the brown dwarf position.

\section{DISCUSSION}

An overlay of a near-infrared image and the integrated intensity in the carbon monoxide (CO $J=2-1$ ) line emission is shown in Figure 1. Two spatially resolved blue- and redshifted CO components are symmetrically displaced on opposite sides of the brown dwarf position, with each lobe about $8^{\prime \prime}$ in size, corresponding to $1000 \mathrm{AU}$ in length. This is similar

\footnotetext{
${ }^{5}$ The Submillimeter Array is a joint project between the Smithsonian Astrophysical Observatory and the Academia Sinica Institute of Astronomy and Astrophysics and is funded by the Smithsonian Institution and the Academia Sinica.
}

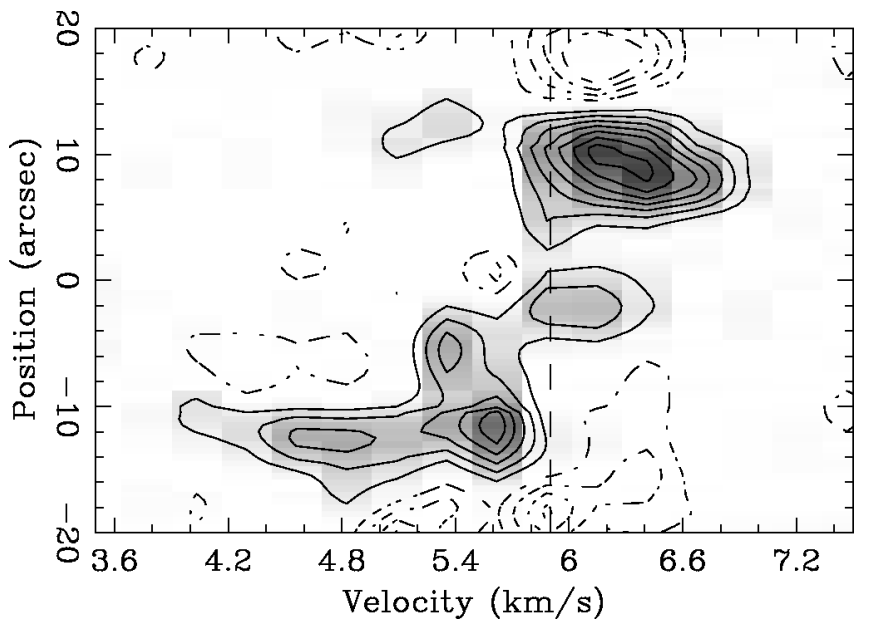

FIG. 2.-Position-velocity (PV) cut diagram for $\mathrm{CO} J=2 \rightarrow 1$ emission at a position angle of $3^{\circ}$. The contours are $-12,-9,-6,-3,3,6,9,12, \ldots$ times the rms of $0.2 \mathrm{Jy}$ beam $^{-1}$. The systemic velocity of the brown dwarf, which is estimated by an average of the velocities of red- and blueshifted components, is indicated by the dashed line. Our value of $5.9 \pm 0.27 \mathrm{~km}$ $\mathrm{s}^{-1}$ is consistent with the previously measured value (Whelan et al. 2005) of $7 \pm 8 \mathrm{~km} \mathrm{~s}^{-1}$ within the error bar. Both blue- and redshifted components show a wide range of the velocity in their structure, which appears to be the bowshock surfaces as observed in young stars (Lee et al. 2000). These surfaces are formed at the head of the jet and accelerate the material in the bow-shock sideways (e.g., Masson \& Chernin 1993).

to the typical pattern of bipolar molecular outflows as seen in young stars (Lada 1985). The two outflow components with a wide range of velocity (Fig. 2) suggest a bow shock structure, an effect of the interaction between the jet propagation and the ambient material, which appears very similar to the bow shock phenomena seen in young stars (Lee et al. 2000). Such a CO outflow morphology suggests that the jet-driven bow shock model (e.g., Masson \& Chernin 1993) may be at work in ISOOph 102.

Following the standard manner (Cabrit \& Bertout 1990; André et al. 1990), we calculate the outflow properties. We use a value of $35 \mathrm{~K}$ (Loren et al. 1983) for the excitation temperature, and we derive a lower limit to the outflow mass of $3.2 \times 10^{-5} M_{\odot}$. If we correct for optical depth with a typical value of 5 (Levreault 1988a), we obtain an upper limit to the outflow mass of $1.6 \times 10^{-4} M_{\odot}$. This value is smaller than the ones observed in young stars (Levreault 1988b) by 3 orders of magnitude.

To estimate the outflow inclination, we combine our SMA data with archival infrared data of the Spitzer Space Telescope. For the infrared photometry, we use the archival basic calibrated data (PIDs: 58, 177) that were reduced with IRAF $^{6}$ to measure flux densities. Disk modeling is performed as in previous works (see Riaz \& Gizis 2008 and references therein). The circumstellar geometry consists of a rotationally flattened infalling envelope, bipolar cavities, and a flared accretion disk in hydrostatic equilibrium. The disk density is proportional to $\varpi^{-\alpha}$, where $\varpi$ is the radial coordinate in the disk midplane and $\alpha$ is the radial density exponent. The disk scale height increases with radius, $h=h_{0}\left(\varpi / R_{*}\right)^{\beta}$, where $h_{0}$ is the scale height at $R_{*}$ (the brown dwarf radius) and $\beta$ is the flaring power. Stellar parameters are obtained from Natta et al. (2004), and

\footnotetext{
${ }^{6}$ IRAF is distributed by the National Optical Astronomy Observatories, which are operated by the Association of Universities for Research in Astronomy, Inc., under cooperative agreement with the National Science Foundation.
} 


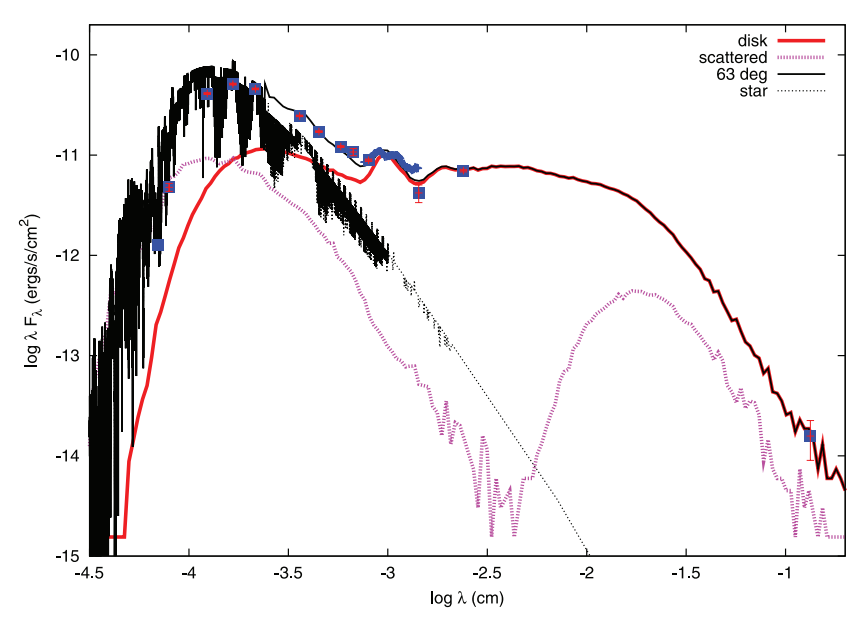

FIG. 3.-Best-fit model for ISO-Oph 102. Contributions from the stellar photosphere, the disk, and the scattered flux are indicated. Optical and nearinfrared data collected from the Vizier database, infrared fluxes from Natta et al. (2002) and measured based on data from the Spitzer archive (IRAC, MIPS, IRS), and millimeter data from our observations are shown.

an accretion rate of $10^{-9} M_{\odot} \mathrm{yr}^{-1}$ is used. The NextGen (Hauschildt et al. 1999) atmosphere file for an effective temperature $T_{\text {eff }}$ of $2700 \mathrm{~K}$, and $\log g=3.5$, where $g$ is the surface gravity, is used to fit the atmosphere spectrum of the central substellar source. The disk shows some flaring longward of $\sim 10 \mu \mathrm{m}$, and the best model fit was obtained using a disk mass of $8 \times$ $10^{-3} M_{\odot}$, an inclination angle between $63^{\circ}$ and $66^{\circ}$, an outer disk radius of $80 \mathrm{AU}$, and an inner disk radius equal to $R_{\mathrm{sub}}$, where $R_{\text {sub }}$ is the dust sublimation radius: $1 R_{\text {sub }} \sim 6.8 R_{\odot}$. There is thus no inner hole in the disk since it would have to be larger than $R_{\text {sub }}$. The outer disk radius and the disk mass are well constrained by the millimeter observations. A more face-on inclination can be ruled out as it results in larger-thanobserved mid- and far-infrared fluxes. Figure 3 presents the best fit from the disk modeling obtained using a disk mass of $8 \times 10^{-3} M_{\odot}$, an inclination angle between $63^{\circ}$ and $66^{\circ}$, and an outer disk radius of $80 \mathrm{AU}$, resulting in a projected disk radius of 32-36 AU. This immediately explains the nondetection of the redshifted optical jet component, estimated to be about $15 \mathrm{AU}$ in length as reported in the previous observation (Whelan et al. 2005). Since the projected disk radius is larger than the jet length, the disk hides the redshifted jet component from our view.

We use the observed maximum flow velocity of $2.2 \mathrm{~km}$ $\mathrm{s}^{-1}$ and apply a correction for the outflow inclination to compute upper limit values for the kinematic and dynamic parameters. We find that the momentum is $P=1.4 \times 10^{-4} M_{\odot} \mathrm{km} \mathrm{s}^{-1}$, the energy is $E=3.1 \times 10^{-4} M_{\odot} \mathrm{km}^{2} \mathrm{~s}^{-2}$, the force is $F=$ $5.9 \times 10^{-8} M_{\odot} \mathrm{km} \mathrm{s}^{-1} \mathrm{yr}^{-1}$, and the mechanical luminosity is $L=2.1 \times 10^{-5} L_{\odot}$, where $L_{\odot}$ is the solar luminosity. A correction for the optical depth factor of 5 and a missing flux factor of 3 for SMA (Bourke et al. 2005) will increase these upper limit values by a factor of 15 . Using the lower value of the outflow mass and a correction factor of 10 applied for the outflow duration time (Parker et al. 1991), we derive the outflow mass-loss rate of $1.4 \times 10^{-9} M_{\odot} \mathrm{yr}^{-1}$, which is smaller than a typical value for T Tauri stars (Lada 1985) by 2 orders of magnitude. One should note that these values are roughly estimated, depending on the correction factor values used in the calculation. One should also note that the outflow mass and the mass-loss rate values from ISO-Oph 102 appear similar to

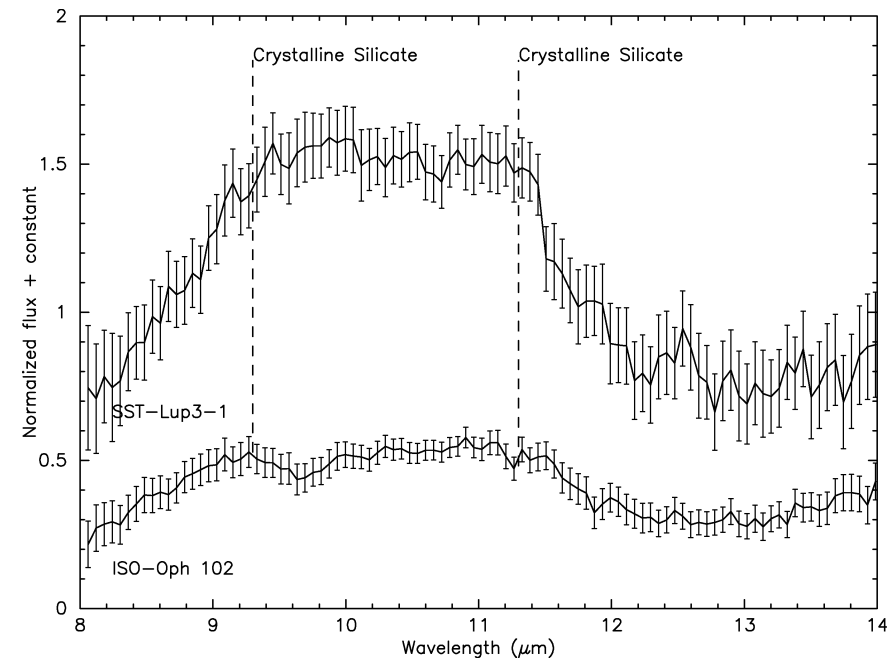

FIG. 4.- Infrared spectral comparison between ISO-Oph 102 and SST-Lup31 (Merín et al. 2007) whose spectrum was obtained from the Spitzer archival data (PID: 179). Continuum subtraction was done following the literature (van Boekel et al. 2003; Apai et al. 2005). The crystalline silicate features at 9.3 $\mu \mathrm{m}$ (mainly enstatite) and $11.3 \mu \mathrm{m}$ (forsterite) are indicated.

that of L1014-IRS (Bourke et al. 2005), suggesting that they share the same origin. However, as clearly stated in Huard et al. (2006), L1014-IRS could be an embedded protostar or a proto-brown dwarf, corresponding to the earliest stages of star or brown dwarf formation processes, respectively. More observations are needed to confirm the L1014-IRS nature and study the connection between ISO-Oph 102 and L1014-IRS in the brown dwarf formation phase.

We also examine the possibility that the emission might be due to bound motions and not outflow emission. This would require an interior mass (Lada 1985) of $2.7 M_{\odot}$ for an outflow size of $1000 \mathrm{AU}$ with a velocity of $2.2 \mathrm{~km} \mathrm{~s}^{-1}$, which is significantly larger than the core mass of $<0.4 M_{\odot}$ within the same radius (Motte et al. 1998; Young et al. 2006). We therefore conclude that the detected emission is from the outflow.

The IRS infrared (7.5-14.3 $\mu \mathrm{m}$; Houck et al. 2004) spectra of ISO-Oph 102, whose basic calibrated data were downloaded from the Spitzer archive (PID: 3499) and reduced using the SMART software package (Higdon et al. 2004), are also analyzed. It is worthy to note here that the mid-infrared spectrum of ISO-Oph 102 shows crystalline silicate features: enstatite $\left(\mathrm{MgSiO}_{3}\right)$ at $9.3 \mu \mathrm{m}$ and very strong forsterite $\left(\mathrm{Mg}_{2} \mathrm{SiO}_{4}\right)$ at $11.3 \mu \mathrm{m}$ (see Fig. 4). The brown dwarf is indeed an analog of SST-Lup3-1, which is an M5.5 brown dwarf in the Lupus III dark cloud (Merín et al. 2007). The crystalline contribution to the silicate feature (flux at $11.3 \mu \mathrm{m}$ over flux at $9.8 \mu \mathrm{m}$ ) in ISO-Oph 102 is 1.1 , even greater than the value of 0.9 in SST-Lup3-1 (Merín et al. 2007). This provides direct evidence of grain growth and dust settling, indicating that the object is in the transition phase between classes II and III (a class with an optically thin disk) and the brown dwarf is reaching the final mass.

\section{SUMMARY}

Here we report the first confirmed detection of the bipolar molecular outflow in young brown dwarfs. We characterize the fundamental properties of the outflow with the first insight on its morphology. The results indicate that the bipolar molecular outflow in brown dwarfs is very similar to outflows as seen in young stars but scaled down by 3 and 2 orders of magnitude 
for the outflow mass and the mass-loss rate, respectively. We demonstrate for the first time that brown dwarfs, even young planetary mass objects (Martín et al. 2001), can launch a bipolar molecular outflow, and hence we confirm that they are likely to have a common origin with the low-mass stars. This suggests that the terminal stellar/brown dwarf mass is not due to different formation mechanisms but more likely due to the initial mass of the cloud core. We also show that there is evidence for dust crystallization in the disk around the brown dwarf. In brown dwarfs the molecular outflows may be longer lived than in lowmass stars, and they coexist with grain growth and crystallization. Outflows from brown dwarfs may play a crucial role in sweeping out gas from the disk and favoring the formation of rocky planets.

N. P.-B. has been aided in this work by a Henri Chrétien International Research Grant administered by the American Astronomical Society. This work is based in part on observations made with the Spitzer Space Telescope, which is operated by the Jet Propulsion Laboratory, California Institute of Technology, under a contract with NASA. This work has made use of the Centre de Données astronomiques de Strasbourg (CDS) database.

\section{REFERENCES}

André, P., Martín-Pintado, J., Despois, D., \& Montmerle, T. 1990, A\&A, 236, 180

Apai, D., Pascucci, I., Bouwman, J., Natta, A., Henning, T., \& Dullemond, C. P. 2005, Science, 310, 834

Bachiller, R. 1996, ARA\&A, 34, 111

Bachiller, R., \& Gómez-González, J. 1992, A\&A Rev., 3, 257

Bourke, T. L., et al. 2005, ApJ, 633, L129

Cabrit, S., \& Bertout, C. 1990, ApJ, 348, 530

de Geus, E. J., de Zeeuw, P. T., \& Lub, J. 1989, A\&A, 216, 44

Fernández, M., \& Comerón, F. 2001, A\&A, 380, 264

Greene, T. P., \& Young, E. T. 1992, ApJ, 395, 516

Hauschildt, P. H., Allard, F., \& Barron, E. 1999, ApJ, 512, 377

Higdon, S. J. U., et al. 2004, PASP, 116, 975

Ho, P. T. P., Moran, J. M., \& Lo, K. Y. 2004, ApJ, 616, L1

Houck, J. R., et al. 2004, ApJS, 154, 18

Huard, T. L., et al. 2006, ApJ, 640, 391

Jayawardhana, R., Mohanty, S., \& Basri, G. 2003, ApJ, 592, 282

Lada, C. J. 1985, ARA\&A, 23, 267

Lee, C.-F., Mundy, L. G., Reipurth, B., Ostriker, E. C., \& Stone, J. M. 2000, ApJ, 542, 925

Levreault, R. M. 1988a, ApJS, 67, 283 1988b, ApJ, 330, 897

Loren, R. B., Sandqvist, A., \& Wootten, A. 1983, ApJ, 270, 620

Luhman, K. L., Joergens, V., Lada, C., Muzerolle, J., Pascucci, I., \& White, R. 2007, in Protostars and Planets V, ed. B. Reipurth, D. Jewitt, \& K. Keil (Tucson: Univ. Arizona Press), 443

Martín, E. L., Dougados, C., Magnier, E., Ménard, F., Magazzù, A., Cuillandre, J.-C., \& Delfosse, X. 2001, ApJ, 561, L195
Martín, E. L., Rebolo, R., \& Magazzù, A. 1994, ApJ, 436, 262

Masson, C. R., \& Chernin, L. M. 1993, ApJ, 414, 230

McKee, C. F., \& Ostriker, E. C. 2007, ARA\&A, 45, 565

Merín, B., et al. 2007, ApJ, 661, 361

Motte, F., André, P., \& Neri, R. 1998, A\&A, 336, 150

Natta, A., Testi, L., Comerón, F., Oliva, E., D’Antona, F., Baffa, C., Comoretto, G., \& Gennari, S. 2002, A\&A, 393, 597

Natta, A., Testi, L., Muzerolle, J., Randich, S., Comerón, F., \& Persi, P. 2004, A\&A, 424, 603

Parker, N. D., Padman, R., \& Scott, P. F. 1991, MNRAS, 252, 442

Pudritz, R. E., Ouyed, R., Fendt, Ch., \& Brandenburg, A. 2007, in Protostars and Planets V, ed. B. Reipurth, D. Jewitt, \& K. Keil (Tucson: Univ. Arizona Press), 277

Raga, A. C., \& Cabrit, S. 1993, A\&A, 278, 267

Reipurth, B., \& Bally, J. 2001, ARA\&A, 39, 403

Riaz, B., \& Gizis, J. E. 2008, ApJ, 681, 1584

Shu, F. H., Ruden, S. P., Lada, C. J., \& Lizano, S. 1991, ApJ, 370, L31

Stassun, K. G., Mathieu, R. D., \& Valenti, J. A. 2006, Nature, 440, 311

van Boekel, R., Waters, L. B. F. M., Dominik, C., Bouwman, J., de Koter, A., Dullemond, C. P., \& Paresce, F. 2003, A\&A, 400, L21

Whelan, E. T., Ray, T. P., Bacciotti, F., Natta, A., Testi, L., \& Randich, S. 2005, Nature, 435, 652

Whelan, E. T., Ray, T. P., Randich, S., Bacciotti, F., Jayawardhana, R., Testi, L., Natta, A., \& Mohanty, S. 2007, ApJ, 659, L45

Whitworth, A., Bate, M. R., Nordlund, Å., Reipurth, B., \& Zinnecker, H. 2007, in Protostars and Planets V, ed. B. Reipurth, D. Jewitt, \& K. Keil (Tucson: Univ. Arizona Press), 459

Young, K. E., et al. 2006, ApJ, 644, 326 